\title{
Awaking and sleeping of a complex network
}

\author{
R. López-Ruiz ${ }^{\mathrm{a}, *}$, Y. Moreno ${ }^{\mathrm{b}}$, A.F. Pacheco ${ }^{\mathrm{b}}$, S. Boccaletti ${ }^{\mathrm{c}}$, D.-U. Hwang ${ }^{\mathrm{c}}$ \\ ${ }^{a}$ Departament of Computer Science and BIFI, Faculty of Sciences, University of Zaragoza, 50009, Zaragoza, Spain \\ ${ }^{\mathrm{b}}$ Departament of Theoretical Physics and BIFI, Faculty of Sciences, University of Zaragoza, 50009, Zaragoza, Spain \\ ${ }^{\mathrm{c}}$ Istituto Nazionale di Ottica Applicata, Largo Enrico Fermi, 6, 50125-Firenze, Italy
}

Received 17 June 2005; accepted 19 April 2006

\begin{abstract}
A network with a logistic-like local dynamics is considered. We implement a mean-field multiplicative coupling among first-neighbor nodes. When the coupling parameter is small, the dynamics is dissipated and there is no activity: the network is turned off. For a critical value of the coupling, a non-null stable synchronized state, which represents a turned on network, emerges. This global bifurcation is independent of the network topology. We characterize the bistability of the system by studying how to perform the transition, which is now topology dependent, from the active state to that with no activity, for the particular case of a scale-free network. This could be a naive model for the wakening and sleeping of a brain-like system, i.e., a multi-component system with two different dynamical behaviors.
\end{abstract}

(C) 2006 Elsevier Ltd. All rights reserved.

Keywords: Complex networks; Neuronal models; Brain-like systems; Logistic coupling; Bistability

\section{Introduction}

Understanding multi-component systems such as the brain is a formidable challenge. A multidisciplinary effort, from medicine, psychiatry, neurobiology and neural computation, is required to enlighten how it works, processes information and takes decisions.

Different models have been proposed to catch the computational principles of mental processes. Neural networks are considered as a paradigmatic model alternative to the more traditional models such as finite automata, Turing machines and Boolean circuits. In fact, neural nets have an inspiration more grounded in the neurophysiological structure of the neuronal system. A survey of the underlying results concerning the computational power and complexity issues of neuronal network models can be found in Sima and Orponen (2003) and references therein.

In a certain sense and from a physical point of view, the brain can be considered as a clock controlled by the internal circadian rhythm. It is synchronized with the day/night cycle (Winfree,

\footnotetext{
* Corresponding author. Tel.: +34 976 761134; fax: +34 976761132 .

E-mail addresses: rilopez@unizar.es (R. López-Ruiz),yamir@unizar.es (Y. Moreno), amalio@ unizar.es (A.F. Pacheco), stefano@ino.it (S. Boccaletti), duhwang@ino.it (D.-U. Hwang).
}

1986). Roughly speaking, two states can be associated with this cycle: awake and asleep. This property is universally observed in all animals. The cerebral activity is dissociated from the sensory and motor neurons in the sleep state. This dissociation is not complete and the brain can still respond to some sensory stimuli. In fact, there are qualitatively different patterns of neural activity between different stages of sleep. Basically, two levels, a deepest and a shallowest, alternate during sleep. The deepest level of sleep is attained rapidly and, as sleep progresses, the average level becomes shallower. The substances that control the connection among neurons monitor these changes in the neural activity, which is formed out of composite states occurring in disconnected brain subdivisions. When the full connections are re-established, the waking state of the brain is recovered (Bar-Yam, 1997).

So, as is suggested by real measurements of electrical brain activity, synchrony seems to be a key concept to explain different aspects of neuronal behavior. The activities of two or more neurons, which we call a functional unit, are said to be synchronized when some kind of temporal correlations exists among them. The conditions for the emergence of these states are a central issue in the research of neuronal activity (Borgers \& Kopell, 2003; Hansel \& Mato, 2003). It has recently been argued (Eguiluz, Chialvo, Cecchi, Baliki, \& Apkarian, 2005) that the distribution of functional connections in the 
human brain follows the same distribution of a scale-free network. This finding means that there are regions in the brain that participate in a large number of tasks while most of the other functional units are only involved in a tiny fraction of the brain's activities. The previous network adds to many examples of such a distribution found in the last few years in fields as diverse as biological, technological and social systems (Bornholdt \& Schuster, 2002; Dorogovtsev \& Mendes, 2003; Pastor-Satorras \& Vespignani, 2004; Strogatz, 2001). These have been termed scale-free networks, because the probability of finding an element with $k$ connections to other elements of the network follows a power law $P(k) \sim k^{-\gamma}$, where $\gamma$ usually lies between 2 and 3 . The absence of a characteristic scale in the connectivity patterns of these latter networks manifests itself in the presence of a few nodes (named hubs) connected to very many nodes, and a larger number of poorly connected nodes. The complex character of the structure of the interactions couples to the dynamical complexity which emerges from the nonlinear character of the interactions, so that, generally, one may say that the Structure-Function correlation problem in real networks has at least two sources of entangled complexity.

In this work, the question of the double (both structural and dynamical) source of entangled complexity is addressed (Llinás, 2003). Specifically, a simple model that can be thought off as an over-simplified model of the sleep-wake cycle of the the brain is proposed. The two basic configurations present in our model, namely the switched on and the switched off states, are formally suggested as a metaphor for the asleep and awake states. How the transition between those states can take place is also indicated. In particular, we show that, as far as bistability is concerned, the underlying structure of the system (structural complexity) is not important. This is in sharp contrast with other results on dynamics on top of complex networks, where the structure radically influences the behavior of the system (Strogatz, 2001). In Section 2, a model for a general network showing bistability is proposed and analyzed. In Section 3, the transition between the active and the inactive states is studied. As our model is thought of as a system made up of functional units, and they seem to be distributed according to a power law, we focus our attention on the on-off transition for the case of a random scale-free network. The last section contains our discussion and conclusions.

\section{The model}

The brain is a complex networked system in which millions of neurons are unidirectionally and locally interconnected (Cajal, 1906). On a larger scale, the brain can be divided into functional modules or regions made up of many neurons. As we have said in the introduction, it is well established that the sleep regime shows phases of high activity, specially the rapid eye movement (REM) stage, but, in the naive picture we outline here, all the sleeping stages are considered to be part of a global phase with a differentiated activity. Thus, a simple approach can consider a functional unit, i.e. a neuron or group of neurons (in the following, neuron or functional unit are used indistinctly), as a discrete dynamical system with two possible states: active (meaning one type of activity) or not (meaning another type of activity). The nonlinear processing of synaptic inputs in cortical neurons has been shown by Kuhn, Aertsen, and Rotter (2004). They studied the response of a model neuron with a simultaneous increase in excitation and inhibition. They found that the firing rate of the model neuron first increases, reaches a maximum, and then decreases at higher input rates. Functionally, this means that the firing rate, commonly assumed to be the carrier of information in the brain, is a non-monotonic function of balanced input. These findings do not depend on details of the model and, hence, are relevant to cells of other cortical areas as well. Hence, in our vision of the brain as a networked system, if $x_{n}^{i}$, with $0<x_{n}^{i}<1$, represents a measurement of the $i$ th functional unit activity at time $n$, it can be reasonable to take the most elemental local nonlinearity, for instance a logistic evolution (May, 1976), which presents a quadratic term, as a first toy-model for the local neuronal activity:

$x_{n+1}^{i}=\bar{p} x_{n}^{i}\left(1-x_{n}^{i}\right)$.

This presents only one stable state for each $\bar{p}$. For $\bar{p}<1$, the dynamics dissipates to zero, $x_{n}^{i}=0$, then it can represent the functional unit with no activity. For $1<\bar{p}<4$, the dynamics is non-null and it would represent an active functional unit. This local transition is controlled by the parameter $\bar{p}$. The functional dependence of this local coupling on the neighboring states is essential in order to get a good brain-like behavior (i.e. as far as the bistability of the sleep-wake cycle is concerned) of the network. As a first approach, we can take $\bar{p}$ as a linear function, depending on the actual mean value, $X_{n}^{i}$, of the neighboring signal activity and expanding the interval $(1,4)$ in the form:

$\bar{p}=p\left(3 X_{n}^{i}+1\right)$,

with

$X_{n}^{i}=\frac{1}{N_{i}} \sum_{j=1}^{N_{i}} x_{n}^{j}$.

$N_{i}$ is the number of neighbors of the $i$ th functional unit, and $p$, which gives us an idea of the interaction of the functional unit with its first-neighbor functional units, is the control parameter. This parameter runs in the range $0<p<p_{\max }$, where $p_{\max } \succeq$ 1. Let us note here that there is an unrealistic bi-directionality in the local neuronal connectivity in this unsophisticated image of brain-like systems. This is not a drawback, since networks with such local dynamics show an interesting bistability that can mimic the brain-like behavior. Hence, the dynamical behavior of these networks present an attractive global null configuration that will be identified as the turned off state of the network. Also, they show a completely synchronized non-null stable configuration that we identify as the turned on state of the network. Therefore, a critical level of noise to transit from the turned off state to the turned on state is needed for a given value of $p$. The different sleep states, including dreams in the human brain, could be interpreted within this panorama as a noisy neuronal activity which does not reach that critical value. The transition from the on state to the off state can be performed 
either by decreasing the coupling $p$ or by making the activity of some units zero. All these dynamical properties are universal for different kinds of local evolution rules of the same type as Eq. (1), the so-called unimodal maps.

Let us mention at this point that phase synchronization and cluster formation in coupled maps on different networks have been studied, for instance, in Jalan and Amritkar (2003). The results exposed in that work are very different from those explained here. Specifically, they found that perfect synchronization leads to clusters with a very small number of nodes. In contrast, robust bistability between two perfectly synchronized states is obtained in our system, as is shown in the next sections.

\subsection{System of two functional units}

Let us start with the simplest case of two interconnected $\left(x^{1}, x^{2}\right)$ functional units. The dynamics (1) is given in this case by the coupled equations:

$x_{n+1}^{1}=p\left(3 x_{n}^{2}+1\right) x_{n}^{1}\left(1-x_{n}^{1}\right)$,

$x_{n+1}^{2}=p\left(3 x_{n}^{1}+1\right) x_{n}^{2}\left(1-x_{n}^{2}\right)$.

Depending on the coupling, $p$ different dynamical regimes are obtained (see the details and nomenclature in references by López-Ruiz and Fournier-Prunaret (2004, 2003)):

- For $0<p<0.75$, the dynamics vanishes. The two functional unit network does not have long-term activity. The whole square $[0,1] \times[0,1]$ of initial conditions shrinks to the turned off configuration, that is, the fixed point $x_{\theta}=$ $(0,0)$.

- For $0.75<p<0.86$, the synchronized state, $x_{+}=(\bar{x}, \bar{x})$, with $\bar{x}=\frac{1}{3}\left\{1+\left(4-\frac{3}{p}\right)^{\frac{1}{2}}\right\}$, which arises from a saddlenode bifurcation for the critical value $p_{0}=0.75$, is a stable turned on state. This state coexists with $x_{\theta}$. The system now presents bistability and, depending on the initial conditions, the final state can be $x_{\theta}$ or $x_{+}$. Switching on the system from $x_{\theta}$ requires a level of noise in both functional units sufficient to render the activity on the basin of attraction of $x_{+}$. Conversely, switching off the two functional unit network can be done, for instance, by making the activity of one functional unit zero, or by making the coupling $p$ lower than $p_{0}$.

- For $0.86<p<0.95$, the active state of the network is now a period-two oscillation. This new dynamical state bifurcates from $x_{+}$for $p=p_{c}=0.86$. A smaller noise is necessary to activate the system from $x_{\theta}$. Making the activity of one functional unit zero continues to be a good strategy to turn off the network.

- For $0.95<p<1$, the active state acquires a new frequency and presents quasiperiodicity. It is still possible to switch off the network by putting one of the functional units to zero.

- For $1<p<1.03$, bistability is lost. When $p=p_{f}=1$, the turned off state, $x_{\theta}$, loses stability and the only stable dynamical state for $p>p_{f}$ is now the turned on network. The network stores the information in a quasiperiodic state.
- For $1.03<p<1.08$, a more complex active state is obtained. In this range, the network can store more complicated information in the stable chaotic state, which is now present in the system.

- For $p>1.0843$, the network loses stability and it cannot store information anymore. Observe that $p_{\max }=1.0843$ in this system.

Let us remark that the system of two functional units exhibits, from a qualitative point of view, the properties that we are looking for in a primitive brain-like system: bistability between an active state and another state with no activity in the range $p_{0}<p<p_{f}$, a necessary noisy level to attain the activation of the network from the switch off state, and two different possible strategies to turn off the system from the active state, by decreasing the coupling under a critical value or by putting one of the functional units to zero.

We proceed now to show that these same properties are still present when the local dynamics of type (1) is mounted on a general complex network.

\subsection{System of many functional units}

The complete synchronization (Boccaletti, Kurths, Osipov, Valladares \& Zhou, 2002) of the network means that $x_{n}^{i}=x_{n}$ for all $i$, with $i=1,2, \ldots, N$ and $N \gg 1$. In this regime, we also have $X_{n}^{i}=x_{n}$. The time evolution of the network on the synchronization manifold is then given by the cubic mapping:

$x_{n+1}=p\left(3 x_{n}+1\right) x_{n}\left(1-x_{n}\right)$.

The fixed points of this system are found by solving $x_{n+1}=x_{n}$. The solutions are $x_{\theta}=0$ and $x_{ \pm}=\frac{1}{3}\left\{1 \pm\left(4-\frac{3}{p}\right)^{\frac{1}{2}}\right\}$. The first state $x_{\theta}$ is stable for $0<p<1$ and $x_{ \pm}$take birth after a saddlenode bifurcation for $p=p_{0}=0.75$. The node $x_{+}$is stable for $0.75<p<1.157$ and the saddle $x_{-}$is unstable. Therefore bistability between the states

$x_{n}^{i}=x_{\theta}, \quad \forall i \longrightarrow$ TURNED OFF STATE,

$x_{n}^{i}=x_{+}, \quad \forall i \longrightarrow$ TURNED ON STATE,

seems to be possible also for $p>p_{0}=0.75$ in the case of many interacting units. But stability on the synchronization manifold does not imply the global stability of it. Small transverse perturbations to this manifold can make the synchronized states unstable. Let us then suppose a general local perturbation $\delta x_{n}^{i}$ of the element activity,

$x_{n}^{i}=x_{*}+\delta x_{n}^{i}$,

with $x_{*}$ representing a synchronized state, $x_{\theta}$ or $x_{+}$. We define the perturbation of the local mean-field as

$\delta X_{n}^{i}=\frac{3}{N_{i}} \sum_{j=1}^{N_{i}} \delta x_{n}^{j}$.

If these expressions are introduced into Eq. (1), the time evolution of the local perturbations are found:

$\delta x_{n+1}^{i}=p\left(3 x_{*}+1\right)\left(1-2 x_{*}\right) \delta x_{n}^{i}+p x_{*}\left(1-x_{*}\right) \delta X_{n}^{i}$. 
The dynamics for the local mean-field perturbation is derived by substituting this last expression in relation (10). We obtain:

$$
\begin{aligned}
\delta X_{n+1}^{i}= & p\left(3 x_{*}+1\right)\left(1-2 x_{*}\right) \delta X_{n}^{i}+3 p x_{*}\left(1-x_{*}\right) \\
& \times \frac{1}{N_{i}} \sum_{j=1}^{N_{i}} \delta X_{n}^{j} .
\end{aligned}
$$

We now express the local mean-field perturbations of the firstneighbors as functions of the local mean-field perturbation $\delta X_{n}^{i}$ by defining the local operational quantity $\sigma_{i}^{n}$ :

$\frac{1}{N_{i}} \sum_{j=1}^{N_{i}} \delta X_{n}^{j}=\sigma_{n}^{i} \delta X_{n}^{i}$,

which is determined by the dynamics itself. If we put together Eqs. (11) and (12), the linear stability of the synchronized states holds as follows:

$$
\begin{aligned}
& \left(\begin{array}{l}
\delta x_{n+1}^{i} \\
\delta X_{n+1}^{i}
\end{array}\right) \\
& =\left(\begin{array}{cc}
p\left(3 x_{*}+1\right)\left(1-2 x_{*}\right) & p x_{*}\left(1-x_{*}\right) \\
0 & p\left(3 x_{*}+1\right)\left(1-2 x_{*}\right)+3 p \sigma_{n}^{i} x_{*}\left(1-x_{*}\right)
\end{array}\right) \\
& \times\left(\begin{array}{c}
\delta x_{n}^{i} \\
\delta X_{n}^{i}
\end{array}\right) .
\end{aligned}
$$

Let us observe that the only dependency on the network topology is included in the quantity $\sigma_{n}^{i}$. The rest of the stability matrix is the same for all the nodes and therefore it is independent of the local and global network organization.

The turned off state is $x_{*}=x_{\theta}=0$. The eigenvalues of the stability matrix are, in this case, $\lambda_{1}=\lambda_{2}=p$. Then, this state is an attractive state in the interval $0<p<1$. It loses stability for $p=1$, then the highest value $p_{f}$ of the parameter $p$ where bistability is still possible satisfies $p_{f} \leq 1$.

The turned on state $x_{+}$verifies $x_{*}=x_{+}=\frac{1}{3}\left\{1+\left(4-\frac{3}{p}\right)^{\frac{1}{2}}\right\}$. If we suppose $\sigma_{n}^{i}=\sigma$, the eigenvalues of the stability matrix are $\lambda_{1}=2-2 p-p\left(4-\frac{3}{p}\right)^{\frac{1}{2}}$ and $\lambda_{2}=\lambda_{1}+\frac{\sigma}{3}\left(3-2 p+p\left(4-\frac{3}{p}\right)^{\frac{1}{2}}\right)$. Let us observe that $\lambda_{1}=-1$ for $p=1$. This implies that the parameter $p_{c}$ for which the synchronized state $x_{+}$loses stability verifies $p_{c} \leq 1$. Depending on the sign of $\sigma$, we can distinguish two cases in the behavior of $p_{c}$ :

- If $0<\sigma<1$, we find that $\left|\lambda_{2}\right|<1$. Then $x_{+}$bifurcates through a global flip bifurcation for $p=p_{c}=1$. In this case, the bifurcation of the synchronized state $x_{+}$for $p_{c}=1$ coincides with the loss of the network bistability for $p_{f}=1$. Hence $p_{c}=p_{f}=1$ for this kind of network, and the bistability holds between $x_{\theta}$ and $x_{+}$in the parameter interval $p_{0}=0.75<p<p_{c}=p_{f}=1$. As an example, an allto-all network shows this behavior, because $\sigma=1$. This is represented in the inset of Fig. 1.

- If $-1<\sigma<0$, then $\lambda_{2}=-1$ is obtained for a $p=p_{c}$ smaller than 1. Therefore it is now possible to obtain an active state different from $x_{+}$in the interval $p_{c}<$ $p<p_{f}$. For instance, simulations show that the global flip bifurcation of the synchronized state for a scale-free network occurs for $p_{c}=0.87 \pm 0.01$. A value of $p=0.866$ is

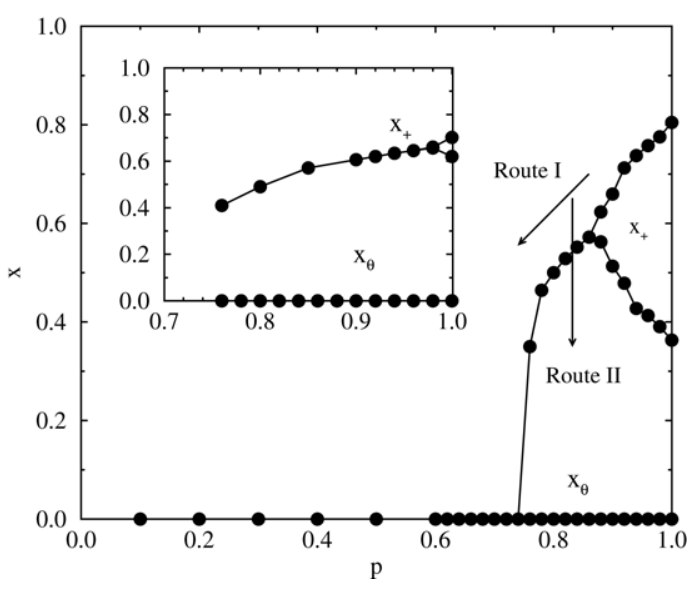

Fig. 1. Stable states $\left(x_{\theta}, x_{+}\right)$of the network for $0<p<1$. Let us observe the two zones of bistability: $p_{0}<p<p_{c}$ and $p_{c}<p<p_{f}$. The main figure corresponds to a scale-free network made up of $N=10^{4}$ elements: $p_{0}=0.75$, $p_{c}=0.87 \pm 0.01$ and $p_{f}=1$. The inset shows the same graph but in an allto-all network of the same size: $p_{0}=0.75, p_{c}=p_{f}=1$. Initial conditions for the $x_{i}$ s were drawn from a uniform probability distribution in the interval $(0,1)$.

obtained from the stability matrix by taking $\sigma=-1$. For this particular network, it is also found that $p_{f}=1$. Then, bistability is possible in the range $p_{0}=0.75<p<p_{f}=$ 1 for this kind of configuration. But now an active state with different dynamical regimes is observed in the interval $p_{c}=0.87<p<p_{f}=1$. If we identify the capacity of information storage with the possibility of the system to access complex dynamical states, then we could assert, in this sense, that a scale-free network has the possibility of storing more elaborate information in the bistable region that an all-to-all network.

Let us note that $\sigma$ also indicates a different behavior of local dissipation, as expression (13) suggests. A positive $\sigma$ means a local in-phase oscillation of the node signal and meanfield perturbations. A negative $\sigma$ means a local out-of-phase oscillation between those signal perturbations. Hence, $\sigma$ also brings some kind of structural network information. In all the cases, the stability loss of the completely synchronized state is mediated by a global flip bifurcation. The new dynamical state arising from that active state for $p=p_{c}$ is a periodic pattern with a local period-two oscillation. The increase in the coupling parameter monitors other global bifurcations that can lead the system towards a pattern of local chaotic oscillations.

\section{Transition between on-off states}

We now proceed to show the different strategies for switching on and off a random scale-free network. The choice of this network is suggested by the recent work (Eguiluz et al., 2005; Buzsàki, Geisler, Henze, \& Wang, 2004) on the distribution of connections among functional units in the brain. They find it to be a power-law distribution. Following this insight, we generate a scale-free network following the Barabási-Albert (BA) recipe (Barabási \& Albert, 1999; Barabási, Albert, \& Jeong, 1999). In this model, starting from 
a set of $m_{0}$ nodes, one preferentially attaches, at each time step, a newly introduced node to $m$ older nodes. The procedure is repeated $N-m_{0}$ times and a network of size $N$ with a power-law degree distribution $P(k) \sim k^{-\gamma}$ with $\gamma=3$ and average connectivity $\langle k\rangle=2 m$ builds up. This network is a clear example of a highly heterogenous network, in that the degree distribution has unbounded fluctuations when $N \rightarrow \infty$. The exponent reported for the brain functional network has $\gamma<3$. However, studies of percolation and epidemic spreading (Callaway, Newman, Strogatz \& Watts, 2000; Moreno, PastorSatorras, \& Vespignani, 2002; Pastor-Satorras \& Vespignani, 2004; Vázquez \& Moreno, 2003) on top of scale-free networks has shown that the results obtained for $\gamma=3$ are consistent with those corresponding to lower values of $\gamma$, with $\gamma>2$. Therefore, we expect that the results shown henceforth are not biased by the use of a different exponent. As explained before, network bistability between the active and inactive states is possible here in the interval $p_{0}=0.75<p<p_{f}=1$ (Fig. 1).

\subsection{Switching off the network}

Two different strategies can be followed to carry the network from the active state to that with no activity (Fig. 1).

- Route I: By doing the coupling $p$ lower than $p_{0}$. This is the easiest and more natural way of performing such an operation. In our naive picture of a brain-like system, it could represent the decrease (or increase, depending on the specific function) of the synaptic substances that provokes the transition from the awake to the asleep state. The flux of these chemical activators is controlled by the internal circadian clock, which is present in all animals, and which seems to be the result of living during millions of years under the day/night cycle.

- Route II: By switching off a critical fraction of functional units for a fixed $p$. Evidently, at first sight, this strategy has no relation to the behavior of a real brain-like system. Thus, this is done by looking over all the elements of the network, and considering that the element activity is set to zero with probability $\lambda$ (which implies that, on average, $\lambda N$ elements are reset to zero). The result of this operation is shown in Fig. 2. Here, the relative size of the biggest (giant) cluster of connected active nodes in the network versus $\lambda$ is plotted for different $p$ s. Note that this procedure does not take into account the existence of connectivity classes, but all nodes are equally treated. The procedure is thus equivalent to simulations of random failure in percolation studies (Callaway et al., 2000). The strategy in which highly connected functional units are first put to zero is more aggressive and leads to quite different results.

Each curve presents three different zones, depending on $\lambda$ :

- the robust phase: For small $\lambda$, the network is stable and only those states put to zero have no activity. There is a linear dependence on the giant cluster size with $\lambda$. In this stage, the switched off nodes do not have the capacity to transmit its actual state to its active neighbors.

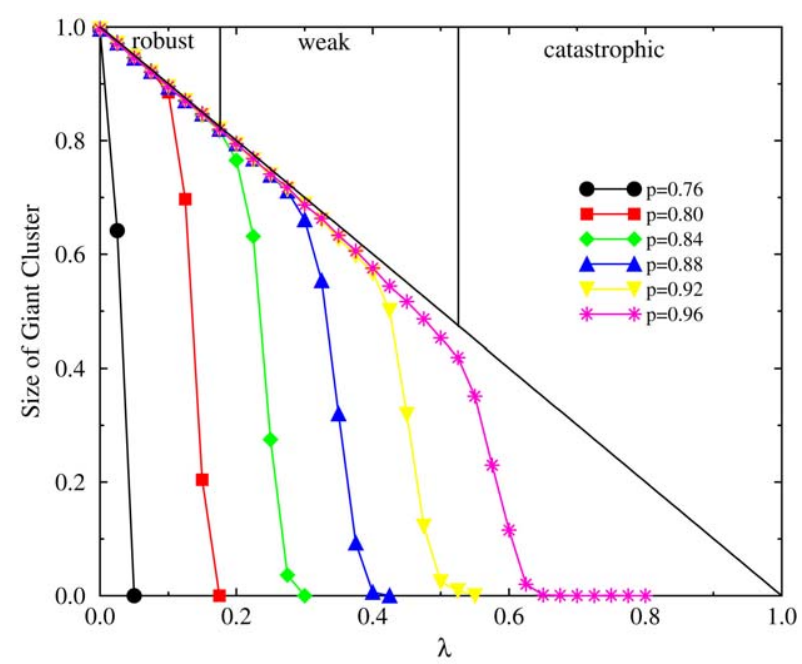

Fig. 2. Turning off a scale-free network. Three different phases in the behavior of the giant cluster size versus $\lambda$ (fraction of switched off nodes) are observed. These three phases are illustrated for $p=0.96$ : the robust phase, the weak phase, and the catastrophic phase (see the text). Other network parameters are as those of Fig. 1.

- the weak phase: For an intermediate $\lambda$, the nodes with null activity can influence its neighborhood and switch off some of them. The linearity between the size of the giant cluster and $\lambda$ shows a higher absolute value of the slope than in the robust zone.

- the catastrophic phase: When a critical $\lambda_{c}$ is reached, the system undergoes a crisis. The sudden drop in this zone means that a small increase of the inactive nodes leads the system to a catastrophe; that is, the null activity is propagated through the whole network and it becomes completely down.

It is worth noticing that, when the system is outside the bistability region for $p>1$, the catastrophic phase does not take place. Instead, the turned off nodes do not spread its dynamical state and the neighboring nodes do not die out. This is because the dynamics of an isolated node is self-sustained when $p>1$. Consequently, we observe that the network breaks down into many small clusters and the transition resembles that of percolation in scale-free nets (Callaway et al., 2000; Vázquez \& Moreno, 2003).

\subsection{Switching on the network}

Two equivalent strategies can be followed for the case of turning on the network (Fig. 3):

- (I) For a fixed $p$, we can increase the maximum value $\epsilon$ of the noisy signal, which is randomly distributed in the interval $(0, \epsilon)$ over the whole system. When $\epsilon$ attains a critical value $\epsilon_{c}$, the noisy configuration can leave the basin of attraction of $x_{\theta}$, whose boundary seems to have the form in phase space of a "hollow cane" around it, and then the network rapidly evolves toward the turned on state;

- (II) If this operation is executed by letting $\epsilon$ be fixed and by increasing the coupling parameter $p$, the final result of 


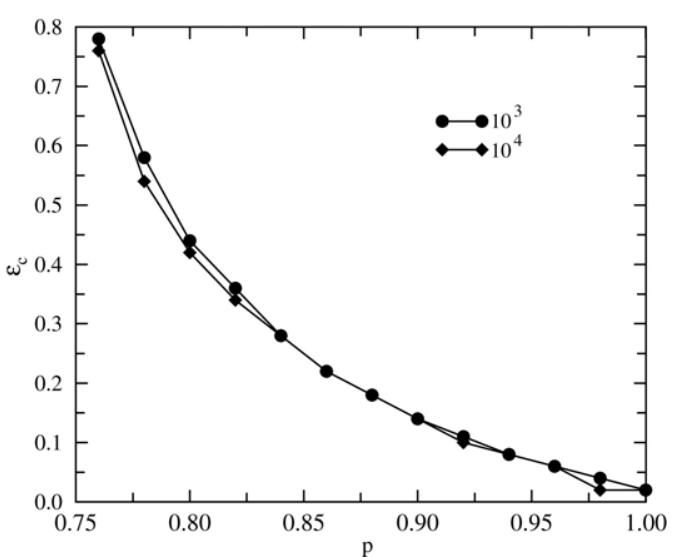

Fig. 3. Turning on a scale-free network. For a fixed $p$, a noisy signal randomly distributed in the interval $(0, \epsilon)$ is assigned to every node. When $\epsilon$ reaches the critical level $\epsilon_{c}$, the network becomes switched on. Other network parameters are as those of Fig. 1.

switching on the network is reached when $p$ takes the value for which $\epsilon=\epsilon_{c}$. The final result is identical in both cases.

Let us remark that the strategy equivalent to the former Route II, that is, the switching on of a critical fraction of functional units, is not possible in this case. It is a consequence of the fact that a switched off functional unit can not be excited by its neighbors and it will maintain indefinitely the same dynamical state $\left(x_{i}=0\right)$.

Finally, let us note that, from Fig. 3, a bigger $p$ requires a smaller $\epsilon_{c}$ to switch on the net. Observe that this behavior could be interpreted in our approach as the smaller level of noise that is needed for waking a brain-like system that it is departing from the sleeping state.

\section{Conclusions}

One of the most challenging scientific problems today is to understand how the millions of neurons of our brain give rise to the emergent property of thinking. Different aspects of neurocomputation touch on this problem: how the brain stores information and how the brain processes it to take decisions or to create new information. Other universal properties of this system are more evident. One of them is the existence of a regular daily behavior: the sleep-wake cycle. The internal circadian rhythm is closely synchronized with the cycle of sun light. Roughly speaking, and depending on the particular species, the brain is awake during the day and it is asleep during the night, or vice versa. Hence, at first sight, this bistable behavior seems not to depend on the precise architecture of the brain.

In this work, we have studied a general network with local logistic dynamics that presents global bistability between an active synchronized state and another synchronized state with no activity. This property is topology and size independent. This is a direct consequence of the local mean-field multiplicative coupling between the first-neighbors. Different routes to transit from one state to the other have been explored for the important case of a scale-free network. If a formal and naive relationship is established between the switched off and switched on states of that network, and the sleep-wake states of a brain, respectively, one would be tempted to assert that this model, regardless of its simplicity, is a good qualitative representation for explaining that specific bistability. Furthermore, on more theoretical grounds, the results obtained here point out the complex interplay between structure and dynamics. While the point at which bistability first appears does not depend on the topology of the underlying network, later bifurcations take place at points that are topology dependent. Additional analysis of the kind followed here might clarify the actual relation between topology and function in networked systems where complex structures coexist with nonlinear dynamics.

\section{Acknowledgments}

Y.M. is supported by MEC through the Ramón y Cajal Program. This work has been partially supported by the Spanish DGICYT Projects FIS2004-05073-C04-01, FIS200500337 and FIS2005-06237. We acknowledge the date 23June-2004 stamped in the preprint of this paper archived in Arxiv.nlin.

\section{References}

Barabási, A. -L., \& Albert, R. (1999). Emergence of scaling in random networks. Science, 286, 509-512.

Barabási, A. -L., Albert, R., \& Jeong, H. (1999). Mean-field theory for scalefree random networks. Physica A, 272, 173-187.

Bar-Yam, Y. (1997). Dynamics of complex systems. Westview Press.

Boccaletti, S., Kurths, J., Osipov, G., Valladares, D. L., \& Zhou, C. S. (2002). The synchronization of chaotic systems. Physics Reports, 366, 1-101.

Borgers, C., \& Kopell, N. (2003). Synchronization in networks of excitatory and inhibitory neurons with sparse, random connectivity. Neural Computation, 15, 509-538.

Bornholdt, S., \& Schuster, H. G. (Eds.) (2002). Handbook of graphs and networks: From the genome to the internet. Berlin: Wiley-VCH.

Buzsàki, G., Geisler, C., Henze, D. A., \& Wang, X. -J. (2004). Interneuron diversity series: Circuit complexity and axon wiring economy of cortical interneurons. Trends in Neurosciences, 27, 186-193.

Cajal, S. R. (1906). The structure and connections of neurons. Nobel Lecture December 12 .

Callaway, D. S., Newman, M. E. J., Strogatz, S. H., \& Watts, D. J. (2000). Network robustness and fragility: Percolation on random graphs. Physical Review Letters, 85, 5468-5471.

Dorogovtsev, S. N., \& Mendes, J. F. F. (2003). Evolution of networks. From biological nets to the internet and the $W W W$. Oxford: Oxford University Press.

Eguiluz, V. M., Chialvo, D. R., Cecchi, G., Baliki, M., \& Apkarian, A. V. (2005). Scale-free brain functional networks. Physical Review Letters, 94, 018102(4).

Hansel, D., \& Mato, G. (2003). Asynchronous states and the emergence of synchrony in large networks of interacting excitatory and inhibitory neurons. Neural Computation, 15, 1-56.

Jalan, S., \& Amritkar, R. E. (2003). Self-organized and driven phase synchronization in coupled maps. Physical Review Letters, 90, 014101(4).

Kuhn, A., Aertsen, Ad., \& Rotter, S. (2004). Neuronal integration of synaptic input in the fluctuation-driven regime. Journal of Neuroscience, 24, 2345-2356.

Llinás, R. R. (2003). The contribution of Santiago Ramón y Cajal to functional neuroscience. Nature Reviews Neuroscience, 4, 77-80. 
López-Ruiz, R., \& Fournier-Prunaret, D. (2004). Complex behavior in a discrete logistic model for the symbiotic interaction of two species. Mathematical Biosciences and Engineering, 1(2), 307-324.

López-Ruiz, R., \& Fournier-Prunaret, D. (2003). Complex patterns on the plane: Different types of basin fractalization in a two-dimensional mapping. International Journal of Bifurcation and Chaos, 13, 287-310.

May, R. M. (1976). Simple mathematical models with very complicated dynamics. Nature, 261, 459-467.

Moreno, Y., Pastor-Satorras, R., \& Vespignani, A. (2002). Epidemic outbreaks in complex heterogeneous networks. The European Physical Journal B, 26, 521-529.
Pastor-Satorras, R., \& Vespignani, A. (2004). Evolution and structure of the internet. Cambridge: Cambridge University Press.

Sima, J., \& Orponen, P. (2003). General-purpose computation with neural networks: A survey of complexity theoretic results. Neural Computation, $15,2727-2778$.

Strogatz, S. H. (2001). Exploring complex networks. Nature (London), 410, 268-276.

Vázquez, A., \& Moreno, Y. (2003). Resilience to damage of graphs with degree correlations. Physical Review E, 67, 015101(R).

Winfree, A. T. (1986). The timing of biological clocks. W.H. Freeman Co.: Scientific American Library. 